\title{
A systematic study on the binding energy of $\Lambda$ hypernuclei
}

\author{
LAN Mi-Xiang ${ }^{1 *}$,LI Lei ${ }^{2 \dagger} \uparrow$ NING Ping-Zhi ${ }^{2 \ddagger}$ \\ 1. Interdisciplinary Center of Theoretical Studies, \\ University of Science and Technology of China, \\ Anhui 230026, P.R.China \\ 2. Department of Physics, Nankai University, \\ Tianjin 300071, P.R.China
}

\begin{abstract}
We calculated the binding energy per baryon of the $\Lambda$ hypernuclei systematically, using the relativistic mean field theory $(\mathrm{RMF})$ in a static frame. Some similar properties are found for most of the $\Lambda$ hypernuclei confirmed by experiments. The data show that a $\Lambda$ hypernucleus will be more stable, if it is made by adding a $\Lambda$ hyperon to a stable normal nuclear core, or by replacing a neutron by a $\Lambda$ hyperon to a stable normal nuclear core. According to our calculations, existences of some new $\Lambda$ hypernuclei are predicted under the frame of RMF.
\end{abstract}

PACS:21.80.+a, 21.10.Dr

The discovery of the strangeness opens a new research field of nuclear physics with strangeness. The research on the structure and property of hypernuclei had produced many meaningful results in 1970s. Some methods, for example, the nuclear core model, RMF and Hartree-Fock-Bogolinbov (HFB), have been used. It has been more than 50 years since the first discovery of a $\Lambda$ hypernuclei by Polish physicists Marion Danysz and Jerzy Pniewski. ${ }^{[1,2]}$ There has been great progress in understanding $\Lambda$ hypernuclei since 1970s in experiment and theory. The hypernuclei properties include the hyperon binding energy $B_{\Lambda}{ }^{[3]}$, the well depth of $\Lambda$-nuclear potential, hypernuclear spins and life times, $\Lambda$ hypernuclei spectroscopy ${ }^{[4]}$, the shrinkage of $\Lambda$ hypernuclear size, $\pi^{0}$ - and $\pi^{+}$-mesonic decays, excited states of hypernuclei and binding energy per baryon, etc have been studied. Up to now, 53 hypernuclei have been found, including 40 single- $\Lambda$ hypernuclei, 3 double- $\Lambda$ hypernuclei, $8 \Xi^{-}$hypernuclei and $2 \Sigma$ hypernuclei ${ }_{\Sigma^{0}}^{4} \mathrm{He},{ }_{\Sigma^{+}}^{4} \mathrm{He}^{[5--9]}$. A lot of experimental data has been accumulated. Systematic studies on the binding energy of $\Lambda$ hypernuclei are required. Sandulescu et al. ${ }^{[10]}$ have used the RMF-BCS approach to treat the resonant states. They considered the resonant states by the scattering states located in the vicinity of the resonance energies, and found that including into the RMF-BCS calculations, only one of these resonant states gets for the neutron radii, and neutron separation energies are practically as same as in the more involved relativistic-Hartree-Bogolinbov (RHB) calculations.

As a convenient method, relativistic mean field(RMF) theory can be used to study nuclear many-body systems. It has been widely adopted in predicting the properties of nuclear matter and finite nuclei, not only normal nuclei but also many kinds of hypernuclei. ${ }^{[11--13]}$ One of the important aspects in RMF is to determine the effective meson-baryon couplings. In this work, we choose NL-SH ${ }^{[14]}$ as the parameters of the meson-baryon coupling constants, which can well describe the properties of finite nuclei and hypernuclei. ${ }^{[11--13]}$ Under this

\footnotetext{
*E-mail: lanbudai@mail.nankai.edu.cn; lan@mail.ustc.edu.cn

$\dagger$ E-mail: lilei@nankai.edu.cn

†E-mail: ningpz@nankai.edu.cn
} 
frame, binding energies per baryon of the ground-state $\Lambda$ hypernuclei is calculated with this uniform set of parameters. After analyzing the theoretical and experimental data with the RMF, some rules can be summarized. Considering the characters of the proved hypernuclei (a $\Lambda$ hypernucleus will be more stable, if it is composed of a $\Lambda$ hyperon adding to a stable normal nuclear core, or a $\Lambda$ hyperon replacing a neutron in a stable normal nuclear core) and the stability of nuclei with magic number and four-periodicity, 71 new $\Lambda$ hypernuclei were predicted, including 5 new hypernuclei whose baryon numbers are smaller than 30 , the other 66 new hypernuclei with a baryon numbers larger than 30. The predicted binding energies imply the possible existence of these new hypernuclei.

In this letter, the binding energies per baryon $(\mathrm{BE} / A)$ of the hypernuclei that have been confirmed by experiments are studied using the RMF method. The $\Lambda$ hypernuclei with baryon number $A<30$ and $A>30$ are considered separately. The results of the RMF are discussed. On basis of the above results, some new hypernuclei are predicted.

First we study the binding energy per baryon of the hypernuclei confirmed by experiments ${ }^{[3,4]}$ from Fig.1, we can see some similar properties with the normal nuclei. When the baryon number is under 30 , the binding energy per baryon present a periodic variety. The binding energy of the medium hypernuclei is larger than those of the light and heavy hypernuclei. The binding energy per baryon of the hypernuclei with the baryon number larger than 120 is around $7.0 \mathrm{MeV}$ and decreases slowly with the increase of the baryon number.

The hypernuclei confirmed by experiments with baryon number under 30 are discussed in the following. For clarity, we plot the binding energy per baryon of some of the hypernuclei with the baryon number under 30 in Fig.2. We can see that the hypernuclei with the baryon number $4 n+1$ are bound more tightly than the nearby ones (except ${ }_{\Lambda}^{9} \mathrm{Be}$ and ${ }_{\Lambda}^{13} \mathrm{C},{ }_{\Lambda}^{10} \mathrm{Be}$ is the most stable hypernuclei among the hypernuclei formed with the normal nuclear core Be. ${ }_{\Lambda}^{14} \mathrm{C}$ is the most stable hypernuclei among the hypernuclei formed with the normal nuclear core C). According to this, we can see hyperon is different from nucleon. If we add a nucleon to a nucleus with the stable structure $4 n$, we will find that the nucleus becomes looser. However, if we add a hyperon to a nucleus with the stable structure, the situation is changed. We can see from the curve that most of the hypernuclei with the baryon number $4 n+1$ are bound more tightly than the hypernuclei with the baryon number $4 n$. Considering that ${ }^{20} \mathrm{Ne},{ }^{24} \mathrm{Mg}$ and ${ }^{28} \mathrm{Si}$ are the nuclei with stable structure $4 \mathrm{n}$ ( $\alpha$ cluster nuclear), we calculate the binding energy per baryon of the ${ }_{\Lambda}^{20} \mathrm{Ne},{ }_{\Lambda}^{21} \mathrm{Ne},{ }_{\Lambda}^{24} \mathrm{Mg},{ }_{\Lambda}^{25} \mathrm{Mg},{ }_{\Lambda}^{29} \mathrm{Si}$, as is listed in Table 1.

The results show that the predicted hypernuclei with baryon number $4 n+1$ are bound more tightly than the hypernuclei with baryon number $4 n$. This is in consistent with what we have found for the hypernuclei confirmed by experiments. $\mathrm{BE} / A_{(\mathrm{th})}$ is the binding energy per baryon calculated by RMF.

We plot the hypernuclei, including both confirmed by experiments and predicted by RMF, with baryon number under 30 in Fig.3. We can see that ${ }_{\Lambda}^{5} \mathrm{He},{ }_{\Lambda}^{17} \mathrm{O}$ are bound more tightly than the nearby hypernuclei. We know that ${ }^{4} \mathrm{He}$ and ${ }^{16} \mathrm{O}$ are not only the $\alpha$ cluster nuclei, but also the nuclei with the double magic number. Here we notice that a magic number nucleus with a $\Lambda$ hyperon is a more stable structure.

For the hypernuclei confirmed by experiments with baryon number larger than 30, we can see from Fig.4 that most of those hypernuclei (except ${ }_{\Lambda}^{32} \mathrm{~S},{ }_{\Lambda}^{33} \mathrm{~S},{ }_{\Lambda}^{56} \mathrm{Fe}$, as is known that $\mathrm{S}$ and Fe nuclei are not the magic number nuclei; however, we know that $\mathrm{S}$ and Fe nuclei are also stable; thus hypernuclei are confirmed by experiments with the $\mathrm{S}$ and Fe normal nuclear core; for the $\mathrm{S}$ nucleus, its baryon number is close to 30 , so we can treat it as the $\alpha$ cluster nucleus) are formed either by adding a $\Lambda$ hyperon to a magic number nuclear core, or by replacing a neutron by a $\Lambda$ hyperon to a magic number nucleus. Following this rule, we predicted some new hypernuclei, as shown in Table 2 and Fig.5

We can see that the variational trend of the binding energy per baryon of the predicted hypernuclei is consistent with that of the hypernuclei confirmed by experiments. The binding energy of the hypernuclei with baryon number between 30 and 65 are nearby $8.8 \mathrm{MeV}$. The binding energy per baryon with baryon number between 87 and 93 are around $7.5 \mathrm{MeV}$. Hypernuclei with the nuclear core $\mathrm{Kr}$ seem more tightly bound, their binding energy per baryon are about $8.7 \mathrm{MeV}$. But the binding energy per baryon of the nearby hypernuclei with the nuclear core $\mathrm{Rb}$ are nearby $7.6 \mathrm{MeV}$. The binding energies per baryon of the hypernuclei with baryon number larger than 111 are around $7.0 \mathrm{MeV}$ and change slowly with the increase 
of baryon number.

Finally, we plot all the hypernuclei, including hypernuclei confirmed by experiments and hypernuclei predicted by RMF, in Fig.6.

In our calculation, the match radius of all the hypernuclei is unchanged. In fact, it differs from the light hypernuclei to the heavy hypernuclei. Here we just give out a range of the errors. BE $/ A_{1}$ refers to the binding energy per baryon with the match radius unchanged. $\mathrm{BE} / A_{2}$ refers to the binding energy per baryon with the match radius being adjusted $\left(\delta_{x}=\right.$ $\left.\mathrm{BE} / A_{1}-\mathrm{BE} / A_{2}, \delta_{x} / \mathrm{x}=\left(\mathrm{BE} / A_{1}-\mathrm{BE} / A_{2}\right) /\left(\mathrm{BE} / A_{1}\right)\right)$.

We can see from Table 3 that when the baryon number is small (under 41 ), the error is 0 . With the increase of the baryon number, the error increases. This shows that our parameters are not very suitable to the medium and heavy hypernuclei. We can see from Table 3 , that the variational trend of the binding energy per baryon of the medium hypernuclei and the heavy hypernuclei with the match radius being adjusted are more like the variational trend of the binding energy per baryon of the normal nuclei.

In summary, among those hypernuclei with the baryon number under $30, \Lambda$ hypernuclei with baryon number $4 n+1$ are bound more tightly than their nearby ones(except ${ }_{\Lambda}^{9} \mathrm{Be}$ and ${ }_{\Lambda}^{13} \mathrm{C}$ ). Most of the $\Lambda$ hypernuclei with the baryon number larger than 30 are composed with the magic number nuclei. From the experimental data, we can see that most of the $\Lambda$ hypernuclei confirmed by experiments are made either by adding a $\Lambda$ hyperon to a stable normal nuclear core, or by replacing a neutron by a $\Lambda$ hyperon to a stable normal nuclear core. Then we have predicted some new $\Lambda$ hypernuclei and studied their binding energies per baryon. The results of RMF show that these predicted new $\Lambda$ hypernuclei are possible to be formed.

\section{References}

[1] Davis D H 2005 Nucl.Phys.A 7543

[2] Danysz M and Pniewski J 1953 Phil.Mag. 44348

[3] Samanta C, Chowdhury P R and Basu D N 2006 Jour.Phys.G:Nucl.Part.Phys. 32363

[4] Hashimoto O and Tamura H 2006 Prog.Part.Nucl.Phys. 57564

[5] Nagae T, Miyachi T, Fukuda T et al 1998 Phys.Rev.Lett. 801605

[6] Outa H, Yamazaki T, Iwasaki M and Hayano R S 1994 Prog.Theor.Phys.Suppl. 117 177

[7] Outa H 1996 Hyperfine interactions. 103227

[8] Hayano R S 1992 Nucl.Phys.A 547 151c

[9] Sawafta R I 1998 Nucl.Phys.A 639 103c

[10] Sandulescu N, Geng L S, Toki H, and Hillhouse G C 2003 Phys.Rev.C 68054323

[11] Tan Yu-Hong, Zhong Xian-Hui, Cai Chong-Hai and Ning Ping-Zhi 2004 Phys.Rev.C 70 054306

[12] Zhong X H, Peng G X, Li L and Ning P Z 2006 Phys.Rev.C 74034321

[13] Zhong X H, Tan Y H, Peng G X, Li L and Ning P Z 2005 Phys.Rev.C 71015206

[14] Sharma M M and Nagarajan M A 1993 Phys.Lett.B 312377 
Table 1: Predicted hypernuclei with baryon number under 30 and their binding energy per particle

\begin{tabular}{llllll}
\hline $\begin{array}{l}\text { Predicted } \\
\text { Hypernuclei }\end{array}$ & $\begin{array}{l}\text { Baryon } \\
\text { number }\end{array}$ & $\begin{array}{l}\mathrm{BE} / A_{(t h)} \\
(\mathrm{MeV})\end{array}$ & $\begin{array}{l}\text { Predicted } \\
\text { Hypernuclei }\end{array}$ & $\begin{array}{l}\text { Baryon } \\
\text { number }\end{array}$ & $\begin{array}{l}\mathrm{BE} / A_{(t h)} \\
(\mathrm{MeV})\end{array}$ \\
\hline${ }_{\Lambda}^{20} \mathrm{Ne}$ & 20 & 7.635 & ${ }_{\Lambda}^{25} \mathrm{Mg}$ & 25 & 7.950 \\
\hline${ }_{\Lambda}^{21} \mathrm{Ne}$ & 21 & 7.714 & ${ }_{\Lambda}^{29} \mathrm{Si}$ & 29 & 8.598 \\
\hline${ }_{\Lambda}^{24} \mathrm{Mg}$ & 24 & 7.723 & - & - & - \\
\hline
\end{tabular}

Table 2: Predicted hypernuclei with baryon number larger than 30 and their binding energy per baryon

\begin{tabular}{llllll}
\hline $\begin{array}{l}\text { Predicted } \\
\text { Hypernuclei }\end{array}$ & $\begin{array}{l}\text { Baryon } \\
\text { number }\end{array}$ & $\begin{array}{l}\mathrm{BE} / A_{(t h)} \\
(\mathrm{MeV})\end{array}$ & $\begin{array}{l}\text { Predicted } \\
\text { Hypernuclei }\end{array}$ & $\begin{array}{l}\text { Baryon } \\
\text { number }\end{array}$ & $\begin{array}{l}\mathrm{BE} / A_{(t h)} \\
(\mathrm{MeV})\end{array}$ \\
\hline${ }_{\Lambda}^{36} \mathrm{~S}$ & 36 & 8.637 & ${ }_{\Lambda}^{112} \mathrm{Sn}$ & 112 & 6.925 \\
\hline${ }_{\Lambda}^{37} \mathrm{~S}$ & 37 & 8.704 & ${ }_{\Lambda}^{113} \mathrm{Sn}$ & 113 & 6.961 \\
\hline${ }_{\Lambda}^{38} \mathrm{Ar}$ & 38 & 8.597 & ${ }_{\Lambda}^{114} \mathrm{Sn}$ & 114 & 6.996 \\
\hline${ }_{\Lambda}^{39} \mathrm{Ar}$ & 39 & 8.728 & ${ }_{\Lambda}^{115} \mathrm{Sn}$ & 115 & 7.032 \\
\hline${ }_{\Lambda}^{48} \mathrm{Ca}$ & 48 & 8.855 & ${ }_{\Lambda}^{116} \mathrm{Sn}$ & 116 & 7.030 \\
\hline${ }_{\Lambda}^{49} \mathrm{Ca}$ & 49 & 8.880 & ${ }_{\Lambda}^{117} \mathrm{Sn}$ & 117 & 7.028 \\
\hline${ }_{\Lambda} \mathrm{Ti}$ & 50 & 8.843 & ${ }_{\Lambda}^{118} \mathrm{Sn}$ & 118 & 7.027 \\
\hline${ }_{\Lambda}^{51} \mathrm{Ti}$ & 51 & 8.904 & ${ }_{\Lambda}^{119} \mathrm{Sn}$ & 119 & 7.026 \\
\hline$\Lambda_{\Lambda}^{52} \mathrm{~V}$ & 52 & 8.909 & ${ }_{\Lambda}^{120} \mathrm{Sn}$ & 120 & 7.026 \\
\hline${ }_{\Lambda}^{52} \mathrm{Cr}$ & 52 & 8.816 & ${ }_{\Lambda}^{121} \mathrm{Sn}$ & 121 & 7.026 \\
\hline${ }_{\Lambda}^{53} \mathrm{Cr}$ & 53 & 8.910 & ${ }_{\Lambda}^{122} \mathrm{Sn}$ & 122 & 7.026 \\
\hline${ }_{\Lambda}^{54} \mathrm{Fe}$ & 54 & 8.774 & ${ }_{\Lambda}^{123} \mathrm{Sn}$ & 123 & 7.027 \\
\hline${ }_{\Lambda}^{55} \mathrm{Fe}$ & 55 & 8.897 & ${ }_{\Lambda}^{124} \mathrm{Sn}$ & 124 & 7.028 \\
\hline${ }_{\Lambda}^{58} \mathrm{Ni}$ & 58 & 8.856 & ${ }_{\Lambda}^{125} \mathrm{Sn}$ & 125 & 7.030 \\
\hline${ }_{\Lambda}^{59} \mathrm{Ni}$ & 59 & 8.847 & ${ }_{\Lambda}^{132} \mathrm{Sn}$ & 132 & 7.066 \\
\hline${ }_{\Lambda}^{60} \mathrm{Ni}$ & 60 & 8.841 & ${ }_{\Lambda}^{133} \mathrm{Sn}$ & 133 & 7.074 \\
\hline${ }_{\Lambda}^{61} \mathrm{Ni}$ & 61 & 8.838 & ${ }_{\Lambda}^{136} \mathrm{Xe}$ & 136 & 6.949 \\
\hline${ }_{\Lambda}^{62} \mathrm{Ni}$ & 62 & 8.839 & ${ }_{\Lambda}^{137} \mathrm{Xe}$ & 137 & 6.968 \\
\hline${ }_{\Lambda}^{63} \mathrm{Ni}$ & 63 & 8.844 & ${ }_{\Lambda}^{138} \mathrm{Ba}$ & 138 & 6.886 \\
\hline${ }_{\Lambda}^{64} \mathrm{Ni}$ & 64 & 8.853 & ${ }_{\Lambda}^{139} \mathrm{Ba}$ & 139 & 6.911 \\
\hline${ }_{\Lambda}^{65} \mathrm{Ni}$ & 65 & 8.865 & ${ }_{\Lambda}^{140} \mathrm{La}$ & 140 & 6.893 \\
\hline${ }_{\Lambda}^{86} \mathrm{Kr}$ & 86 & 8.778 & ${ }_{\Lambda}^{140} \mathrm{Ce}$ & 140 & 6.845 \\
\hline${ }_{\Lambda}^{87} \mathrm{Kr}$ & 87 & 8.796 & ${ }_{\Lambda}^{141} \mathrm{Ce}$ & 141 & 6.874 \\
\hline${ }_{\Lambda}^{87} \mathrm{Rb}$ & 87 & 7.680 & ${ }_{\Lambda}^{141} \mathrm{Pr}$ & 141 & 6.823 \\
\hline${ }_{\Lambda}^{88} \mathrm{Rb}$ & 88 & 7.587 & ${ }_{\Lambda}^{142} \mathrm{Pr}$ & 142 & 6.855 \\
\hline${ }_{\Lambda}^{88} \mathrm{Sr}$ & 88 & 7.657 & ${ }_{\Lambda}^{142} \mathrm{Nd}$ & 142 & 6.800 \\
\hline${ }_{\Lambda}^{89} \mathrm{Sr}$ & 89 & 7.564 & ${ }_{\Lambda}^{143} \mathrm{Nd}$ & 143 & 6.834 \\
\hline${ }_{\Lambda}^{89} \mathrm{Y}$ & 89 & 7.601 & ${ }_{\Lambda}^{144} \mathrm{Sm}$ & 144 & 6.753 \\
\hline${ }_{\Lambda}^{90} \mathrm{Y}$ & 90 & 7.510 & ${ }_{\Lambda}^{145} \mathrm{Sm}$ & 145 & 6.790 \\
\hline${ }_{\Lambda}^{90} \mathrm{Zr}$ & 90 & 7.545 & ${ }_{\Lambda}^{209} \mathrm{~Pb}$ & 209 & 6.726 \\
\hline${ }_{\Lambda}^{91} \mathrm{Zr}$ & 91 & 7.455 & ${ }_{\Lambda}^{210} \mathrm{Bi}$ & 210 & 6.711 \\
\hline${ }_{\Lambda}^{92} \mathrm{Mo}$ & 92 & 7.378 & ${ }_{\Lambda}^{210} \mathrm{Po}$ & 210 & 6.689 \\
\hline${ }_{\Lambda}^{93} \mathrm{Mo}$ & 93 & 7.291 & ${ }_{\Lambda}^{211} \mathrm{Po}$ & 211 & 6.696 \\
\hline & & & & & \\
\hline
\end{tabular}


Table 3: Errors of our calculation

\begin{tabular}{ccccc}
\hline Hypernuclei & $\mathrm{BE} / A_{1}(\mathrm{MeV})$ & $\mathrm{BE} / A_{2}(\mathrm{MeV})$ & $\delta_{x}$ & $\delta_{x} / x$ \\
\hline${ }_{\Lambda}^{10} \mathrm{Be}$ & -6.029 & -6.029 & 0 & 0 \\
\hline${ }_{\Lambda}^{12} \mathrm{~B}$ & -7.184 & -7.184 & 0 & 0 \\
\hline${ }_{\Lambda}^{12} \mathrm{C}$ & -6.937 & -6.937 & 0 & 0 \\
\hline${ }_{\Lambda}^{13} \mathrm{C}$ & -7.801 & -7.801 & 0 & 0 \\
\hline${ }_{\Lambda}^{28} \mathrm{Si}$ & -8.294 & -8.294 & 0 & 0 \\
\hline${ }_{\Lambda}^{41} \mathrm{Ca}$ & -8.744 & -8.744 & 0 & 0 \\
\hline${ }_{\Lambda}^{51} \mathrm{~V}$ & -8.832 & -8.792 & -0.04 & 0.0045 \\
\hline${ }_{\Lambda}^{89} \mathrm{Y}$ & -7.601 & -8.825 & 1.224 & -0.1610 \\
\hline${ }_{\Lambda}^{139} \mathrm{La}$ & -6.866 & -6.866 & 0 & 0 \\
\hline${ }_{\Lambda}^{208} \mathrm{~Pb}$ & -6.721 & -5.850 & -0.871 & 0.1296 \\
\hline
\end{tabular}

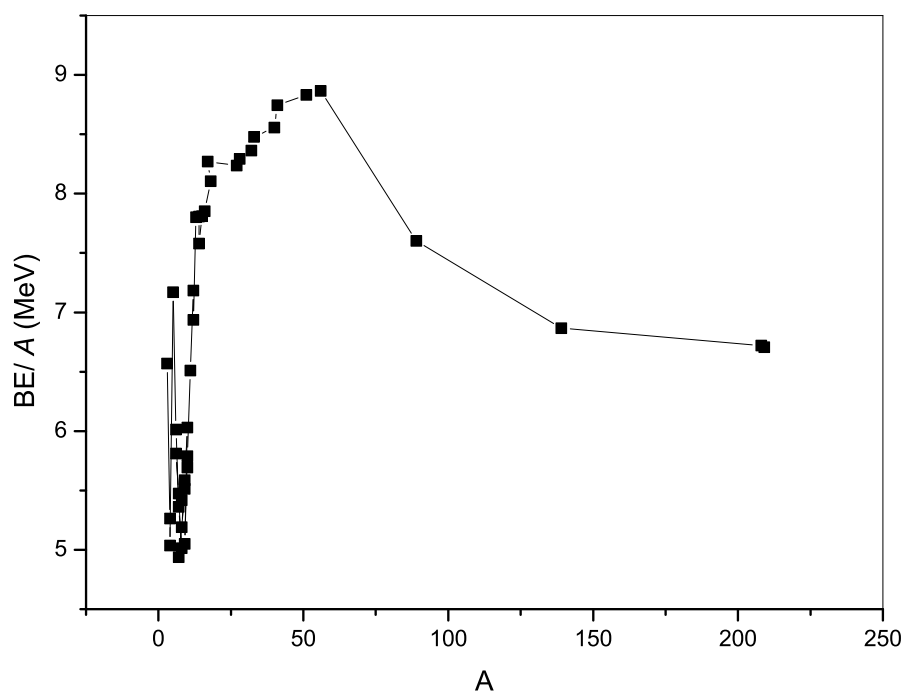

Fig. 1: Binding energy per baryon of the hypernuclei confirmed by experiments 


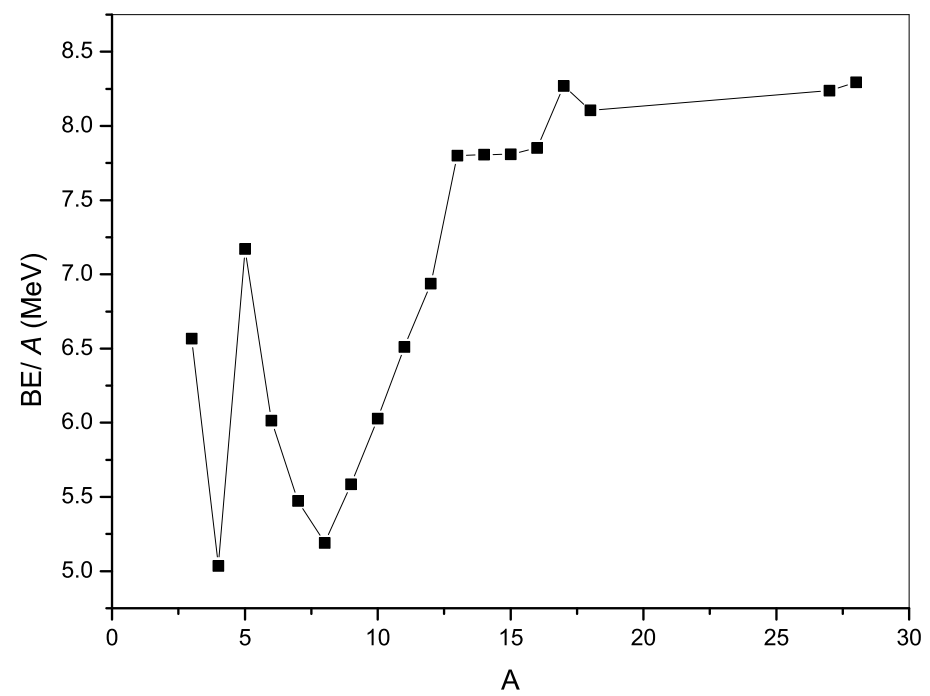

Fig. 2: Binding energy per baryon of some hypernuclei with baryon number under 30

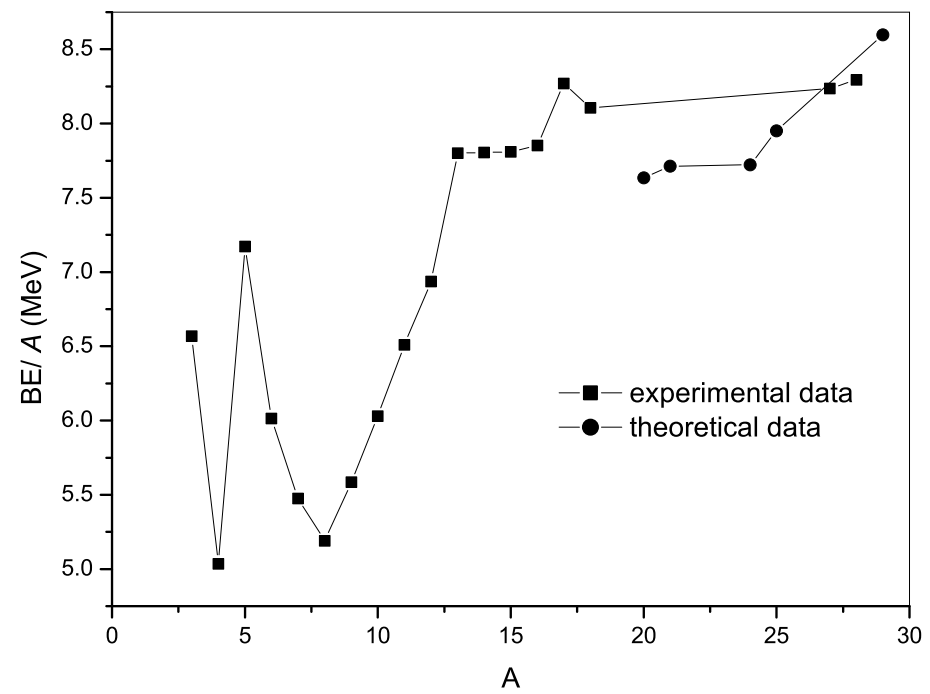

Fig. 3: Binding energy per baryon of some hypernuclei confirmed by experiments and all the predicted hypernuclei with the baryon number under 30 


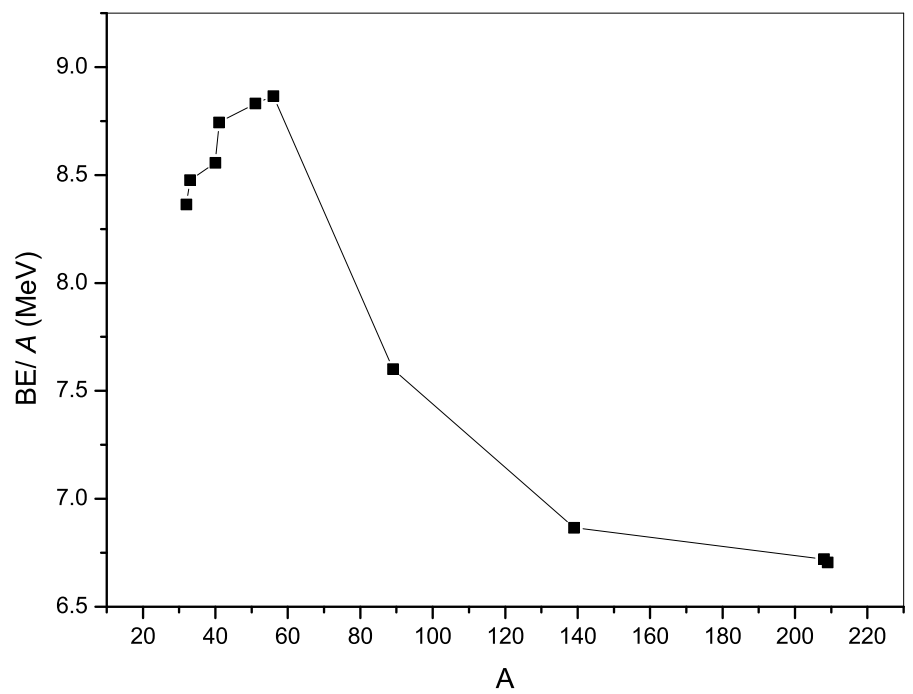

Fig. 4: Binding energy per baryon of the hypernuclei confirmed by experiments

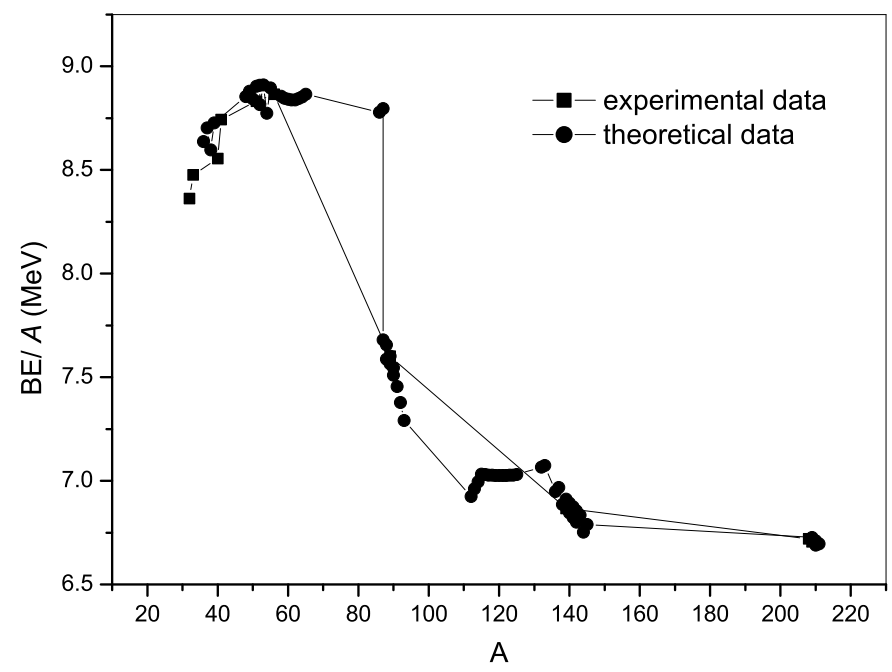

Fig. 5: Binding energy per baryon of the hypernuclei with the baryon number larger than 30 


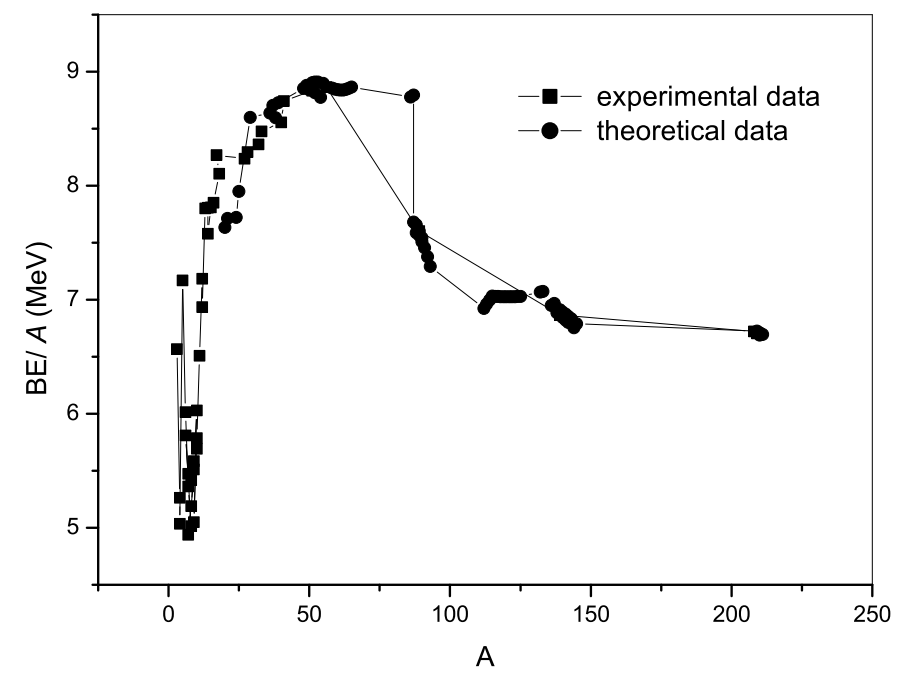

Fig. 6: Binding energy per baryon of the hypernuclei confirmed by experiments and predicted by RMF 